\title{
THE EFFECT OF BLOOD WITHDRAWALS ON CEREBRAL OXYGENATION AND HEMODYNAMICS IN NEWBORNS TREATED WITH VA-ECMO
}

\author{
L. Gerrits ${ }^{1}$, A. De $\mathrm{Mol}^{2}$, A. Van Heijst ${ }^{1}$, D. Liem ${ }^{1}$ \\ ${ }^{1}$ Pediatrics, Radboud University Nijmegen Medical Centre, Nijmegen, ${ }^{2}$ Pediatrics, Albert Schweitzer \\ Hospital, Dordrecht, The Netherlands
}

Background and aims: Veno-arterial extracorporeal membrane oxygenation (va-ECMO) is a rescue therapy for neonates with severe respiratory insufficiency. An important complication is intracranial hemorrhage (ICH). In previous studies we demonstrated that bladderbox alarms during va-ECMO resulted in significant changes of mean arterial blood pressure (MABP), cerebral blood flow (CBF) and cerebral blood volume (CBV). Aim of this study was to determine the effect of blood withdrawals on the occurrence of bladderbox alarms, cerebral oxygenation and hemodynamics.

Methods: In this prospective observational study in newborns $(n=11)$ blood withdrawals performed during the first 24 hours of va-ECMO were studied using near infrared spectrophotometry. Based on concentration changes of cerebral oxyhemoglobin $\left(\mathrm{cO}_{2} \mathrm{Hb}\right)$ and deoxyhemoglobin $(\mathrm{cHHb})$ changes in hemoglobin oxygenation index $\left(\mathrm{cHbD}=\mathrm{cO}_{2} \mathrm{Hb}-\mathrm{cHHb}\right)$ and total hemoglobin ( $\mathrm{ctHb}=\mathrm{cO}_{2} \mathrm{Hb}+\mathrm{cHHb}$ ) were calculated, reflecting changes in $\mathrm{CBF}$ and $\mathrm{CBV}$ respectively. Physiologic parameters and bladderbox alarms were continuously measured.

Results: 133 blood withdrawals were analyzed. Small volume blood withdrawals $(<5 \mathrm{~mL})$ did not result in bladderbox alarms or significant changes in $\mathrm{cHbD}$ or ctHb. On the contrary, $40 \%$ of large volume blood withdrawals (range 5-9 $\mathrm{mL}, \mathrm{n}=10$ ) resulted in bladderbox alarms and a significant decrease of MABP and $\mathrm{cHbD}$.

Conclusions: Large volume blood withdrawals during va-ECMO resulted in bladderbox alarms and (consecutive) changes in cerebral oxygenation and hemodynamics. This might be a risk factor for ICH. We recommend to avoid bladderbox alarms due to large volume blood withdrawals. Small volume blood withdrawals, which are frequently performed in ECMO patients, do not effect cerebral oxygenation and hemodynamics. 